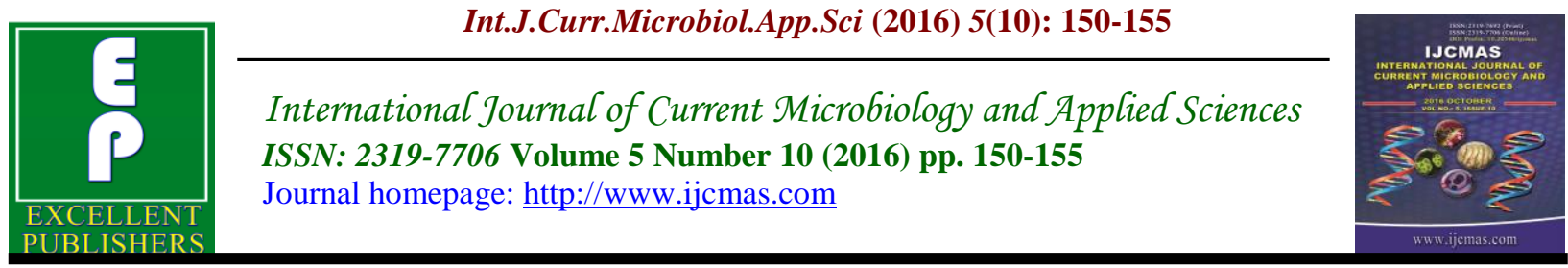

Original Research Article

http://dx.doi.org/10.20546/ijcmas.2016.510.017

\title{
Comparative Evaluation Studies of CSF ADA Activities in Different Neurological Deficit Groups of Patients with Special Emphasis on Meningitis in Indian Scenario
}

\author{
K. Goswami ${ }^{*}$, S. Mookherjee ${ }^{2}$, K. Sridhar ${ }^{3}$, M. Narayanan ${ }^{3}$ and P. Pal ${ }^{4}$ \\ ${ }^{1}$ Department of Biochemistry, Lincoln University College, Kuala Lumpur, Malaysia \\ ${ }^{2}$ North City Diagnostics, Kolkata, West Bengal, India \\ ${ }^{3}$ Meril Diagnostics Pvt. Ltd, Vapi, Gujarat, India \\ ${ }^{4}$ Department of Pharmacology, IIMSAR \& Dr. B C Roy Hospital, Haldia, West Bengal, India \\ *Corresponding author
}

\section{Keywords \\ CSF, \\ ADA, \\ Tubercular \\ Meningitis \\ (TBM).}

\section{Article Info}

Accepted:

12 September 2016

Available Online:

10 October 2016
A B S T R A C T

Tubercular Meningitis (TBM) is an endemic disease in developing countries. Adenosine deaminase activity (ADA) has been of great importance for many years in TBM diagnosis. The present study has been undertaken to assess the levels of CSF-ADA activity in different types of meningitis patients, diagnosed as per clinical and other findings (CSF- Cell Count and Types and.CSF Protein and Sugar content) in comparison to Control group, who are to be considered as other than those with meningitis based on clinical and CSF studies. In total, 98 CSF samples were collected from different groups of patients comprising different types of meningitis (Aseptic, Pyogenic, Tubercular, Fungal and Control groups, consisting of 22 patients in each group excepting Fungal Meningitis group having 10 patients. The control group consisted of 22 people without Meningitis. CSF ADA activity was found to be significantly higher (30 IU/L with a mean of $20.99 \pm 4.66$ ) in tubercular meningitis in comparison to other groups of meningitis. Other cases \& the control group showed ADA concentration of $1.36 \pm 0.59 \mathrm{IU} / \mathrm{L}$. The present Study also reveals that CSF ADA concentration was found to be $\leq 5 \mathrm{IU} / \mathrm{L}$ in control group.

\section{Introduction}

Tubercular Meningitis (TBM) is an endemic disease in developing countries, more in low socio-economic status ${ }^{1}$. Though India is the second most populous country in the world, it shows more newly diagnosed TBM cases annually than any other country. There is an urgent need to improve methods for early diagnosis.
A few tests are available for the diagnosis of TBM, such as Adenosine deaminase activity (ADA) (EC 3.5.4.4). ADA has been of interest for many years in TBM. ADA was first isolated from calf mucosa in the early $1940^{2}$. ADA is ubiquitously present in the body, but especially in lymphoid tissue, levels are particularly high in active $\mathrm{T}$ 
Lymphocytes, hence it is associated with disorders that induce $\mathrm{T}$-cell mediated immune responses. It has been shown to be value in the distinction of tubercular pleural effusions ${ }^{3}$. ADA assay may be useful in confirming TBM, but raised levels may also be seen in other Central Nervous System (CNS) disorders (Sarcoidosis, Meningeal lymphoma, Subarachnoid haemorrhage, Neurobrucellosis) rendering it too nonspecific $^{4}$. CSF ADA is thought to be released by T-Lymphocyte during cell mediated immune response in tuberculoses infection.

The objective of the present study was to measure CSF ADA activity in assessing its future possibility as a reliable marker for Meningitis, in addition to conventional parameters of CSF Studies in differentiating tuberculoses from non tuberculoses meningitis.

\section{Materials and Methods}

The study design was cross sectional. The information was gathered from those patients whose CSF fluid sample was sent for ADA. All the Patients presenting with headache, fever, nausea, vomiting, neck rigidity signs of meningitis like Kernigs or Brudzinski's sign, altered sensorium, any focal neurological deficit, cranial nerve palsies, hemiparesis, seizures and /or signs of cerebral dysfunction ranging from confusion, delirium, declining level of sensorium from lethargy to coma, were considered as the cases for the study.

Informed consent was taken from all patients and ethical committee clearance was obtained.

Fresh CSF samples were collected under aseptic precautions in three parts. First part processed for diagnosing meningitis by noting pressure, cell type and count, glucose and protein levels. Second part of CSF for bacterial and fungal culture along with AFB staining. Negative staining for Cryptococcus. Third part of CSF for ADA estimation. The different types of meningitis patients were separated on the basis of CSF Cytochemistry.

TBM diagnosed by average CSF protein conc. $>40 \mathrm{mg} / \mathrm{dl}$, cell count $>50 \mathrm{WBC}$ /cumm predominantly consisting of $>90 \%$ lymphocytes, sugar $>70 \mathrm{mg} / \mathrm{dl} \& \mathrm{CSF}$ culture and or zn staining have revealed AFB.

Pyogenic Meningits is characterized by CSF cell count > 100 WBC/cu.mm predominantly consisting of $\geq 90 \%$ polymorphs having neutrophilic pleocytosis. CSF protein $>40 \mathrm{mg} / \mathrm{dl} \&$ sugar $\leq 30 \mathrm{mg} / \mathrm{dl} \&$ CSF appearance were cloudy or turbid. CSF-culture and gram staining shows may be gram-positive or gram-negative bacteria.

Aseptic meningitis diagnosed by protein $\leq$ $40 \mathrm{mg} / \mathrm{dl}$, cell count> $100 \mathrm{WBC} / \mathrm{cu} . \mathrm{mm}$, sugar $\leq 20 \mathrm{mg} / \mathrm{dl}$ of plasma and consisted predominantly of lymphocytes.

Fungal meningitis diagnosed by normal or slight elevation in pressure, protein $>40$ $\mathrm{mg} / \mathrm{dl}$, cell count $>50 \mathrm{WBC} / \mathrm{cu} . \mathrm{mm}$, Sugar $>70 \mathrm{mg} / \mathrm{dl}$. Fungal culture and staining revealed Cryptococcus.

Critical Patients having initial loss of sensorium with a Clinical sign of hemiparesis, Hemiplegic, Paraplegic or quadriplegic were treated as a control group.

Patients were segregated in to five groups according to diagnosis on the basis of clinical examination and CSF Cytochemistry.

Group A: $\quad$ Tuberculous Meningitis (TBM) 
Group B:

Group C:

Group D:

Group E:
Pyogenic Meningitis

Aseptic Meningitis

Fungal Meningitis

Control (Patients with

non-neurological

disease)

Fresh samples of CSF were obtained by lumber puncture and collected in heparinised vial and subjected for ADA estimation in CSF by spectrophotometric method using Meril Autoquant 400 autoanalyser at optimum wavelength of $546 / 800 \mathrm{~nm}$ and the temperature of $37^{\circ} \mathrm{C}$ as per the modified method of Giusti and Galauti ${ }^{5}$.

CSF ADA activity was photo metrically precisely measured at $546 / 800 \mathrm{~nm}$ in an automated bio chemistry analyzer-Meril Autoquant 400 in avoiding human error.

\section{Results and Discussion}

Total number of patients presented in our studies was 98. Among 98 patients, most common type of meningitis was TBM. The females are preponderance in this study. Also, most commonly affected age group was $23-42$ years followed by children below
10 years.

The common clinical presentation of patients with meningitis in our study were neck rigidity, positive Kering's sign, fever, headache, nausea and vomiting. Also, few patients of meningitis were having focal neurologic deficit, altered sensorium and seizures.

The level of ADA in cerebrospinal Fluid (CSF) was estimated among the different types of meningitis. The observation was depicted in Table1 along with mean and standard deviation $(\mathrm{SD})$ values.

The Upper Table 1 shows that the range of ADA was 6.5 to 30.3 (IU/l) in tubercular meningitis with a mean of $20.99 \pm 4.66$ (IU/l), while a range between 2.8 to 14.3 (IU/l) with a mean of $10.03 \pm 2.5$ (IU/l) was noted in pyogenic meningitis. Similarly, ADA levels between 1.9 to 10.3 (IU/l) with a mean of $5.21 \pm 2.39$ were observed in aseptic group of meningitis and 2.9 to 10.1(IU/l) with a mean of $5.5 \pm 5.0$ were noted in fungal group of meningitis patients. In control group of patients the CSF ADA activity ranges between 0.2-2.5 IU/l with a mean of $1.36 \pm 0.59$.

Table.1 CSF-ADA levels in various groups

\begin{tabular}{|c|l|c|c|}
\hline $\begin{array}{c}\text { Group } \\
\text { Name }\end{array}$ & \multicolumn{1}{|c|}{ Study Group } & Range of ADA( in IU/l) & Mean \pm SD \\
\hline Group A & $\begin{array}{l}\text { Tuberculosis } \\
\text { Meningitis (n=22) }\end{array}$ & $6.5-30.3$ & $20.99 \pm 4.66$ \\
\hline Group B & $\begin{array}{l}\text { Pyogenic Meningitis } \\
(\mathrm{n}=22)\end{array}$ & $2.8-14.3$ & $10.03 \pm 2.5$ \\
\hline Group C & $\begin{array}{l}\text { Aseptic Meningitis } \\
(\mathrm{n}=22)\end{array}$ & $1.9-10.3$ & $5.21 \pm 2.39$ \\
\hline Group D & $\begin{array}{l}\text { Fungal Meningitis } \\
(\mathrm{n}=10)\end{array}$ & $2.9-10.1$ & $5.5 \pm 5.0$ \\
\hline Group E & $\begin{array}{l}\text { Control } \\
(\mathrm{n}=22)\end{array}$ & $0.2-2.5$ & $1.36 \pm 0.59$ \\
\hline
\end{tabular}


Correlation Studies among Different Groups of Meningitis patients vs. Control Groups were undertaken \& represented in the Fig: 1-3A.

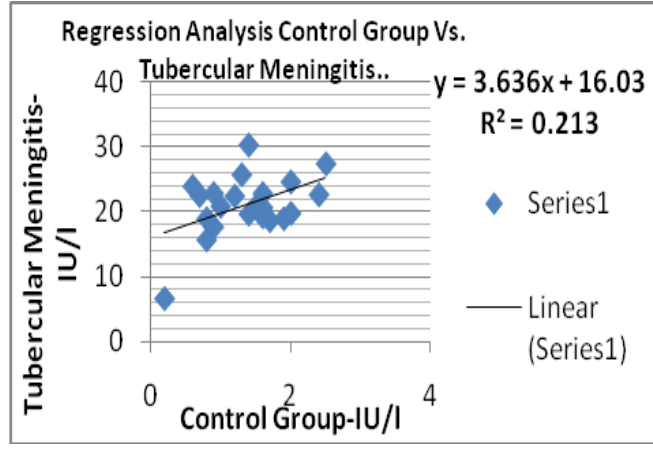

Fig: 1

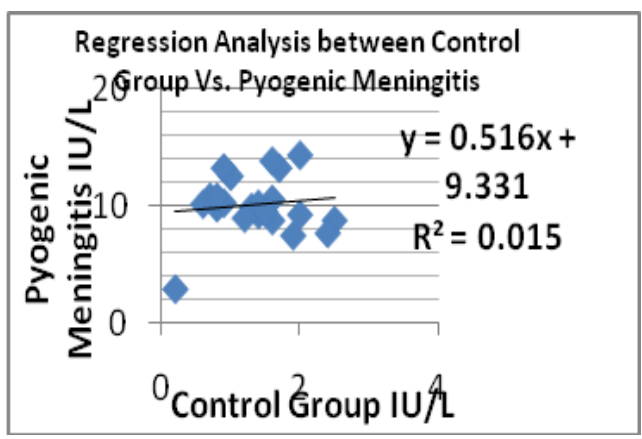

Fig: 2

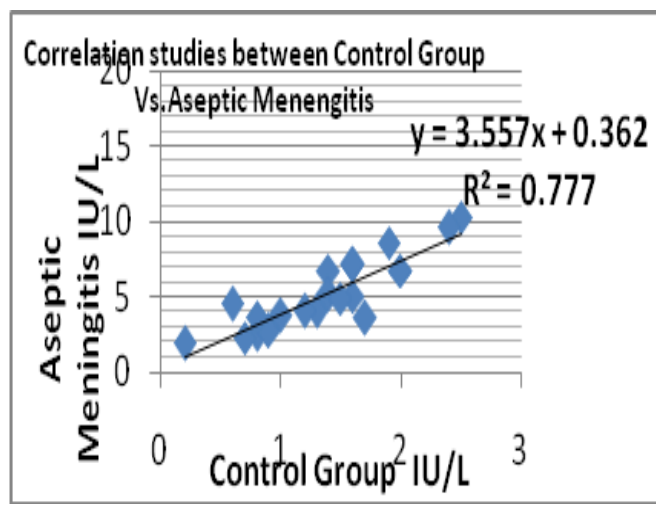

Fig: 3

Both Correlation \& Point of Agreement studies reveals the following facts:

1) In Tubercular meningitis, in majority data points of CSF ADA activity were homosedestically distributed around the regression line around \& above $20 \mathrm{IU} / 1$ (Fig: 1 \& 1A).

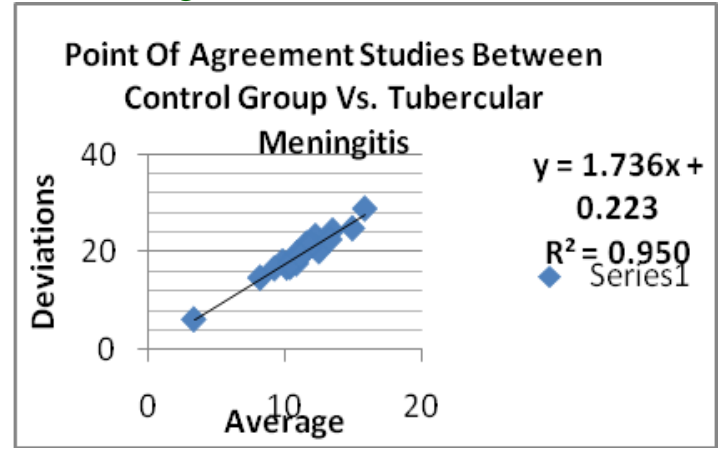

Fig: 1A

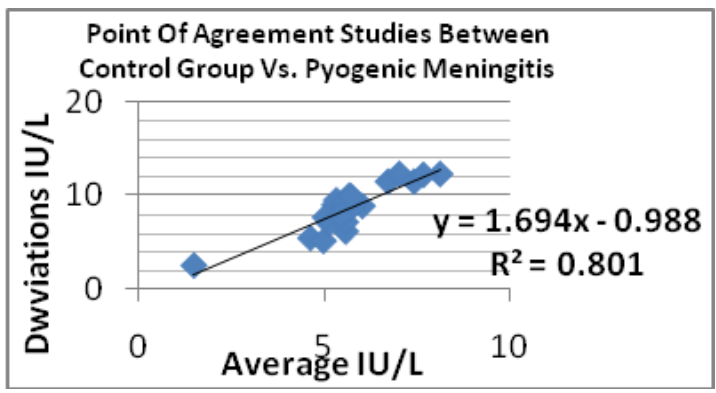

Fig: 2A

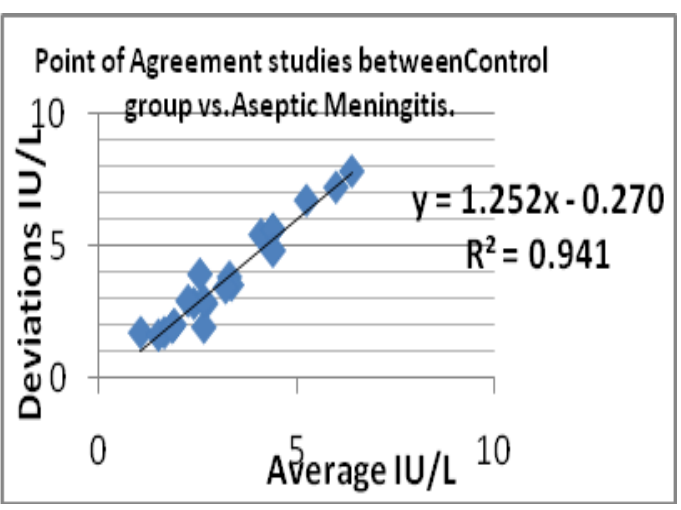

Fig: 3A

2) In pyogenic meningitis, CSF ADA activities were distributed around \& above $10 \mathrm{IU} /$, excepting in one case (Fig: 2 \& 2A).

3) It is interesting to note that, in both aseptic \& Fungal meningitis, CSF ADA activities were homosedestically 
distributed around $5 \mathrm{IU} / \mathrm{l}$ or above along the regression line in majority cases (approx 19patients out of total 22 patients) in comparison to control groups, suggesting no appreciable change in activities indicating the future possibilities of Cut-off range of CSFADA activities should be around < 5 IU/l in Indian population (Fig: 3).

4) Point of agreement studies between control vs. Aseptic meningitis groups also reveals that difference of CSF ADA activities were restricted below < 5 IU/l while average of activities lies < 5 in majority cases (excepting in 3 cases) indicating a positive correlation with approx. sensitivity of $>90 \%$ ( Fig; $3 \& 3 \mathrm{~A})$.

Diagnosis of tubercular Meningitis is quiet difficult with AFB staining which is less sensitive. We can't differentiate accurately tubercular meningitis from other types of infections meningitis with routine CSF laboratory parameters. Accurate diagnosis of tubercular meningitis is needed for early treatment.

ADA estimation in CSF was reported to be useful in diagnosing tubercular meningitis and can differentiate TBM from normal subject or patients with other neurological disorders (Blake et al., 1982). ADA estimation in CSF is a useful tools to differentiate TBM (Malan et al., 1984). Also, other researchers have also observed the usefulness of ADA activity in CSF in the diagnosis of tubercular meningitis (Mishra $e t$ al., 1995).

In the present study with ADA activity levels in CSF's cutoff value should around < $5 \mathrm{IU} / \mathrm{l}$ with approx .sensitivity $>90 \%$ and Specificity around $85 \%$ for the diagnosis of tubercular meningitis. The cut-off level of
CSF ADA activity were noted around or > 20 IU/l in tubercular meningitis in our studies. CSF ADA activities (around more than $20 \mathrm{IU} / \mathrm{l}$ ) were 4 times greater in Tubercular meningitis comparing with other groups of all meningitis with normal cut-off value approx. $<5 \mathrm{IU} / \mathrm{l}$.

The average cut-off value of CSF ADA level is $10 \mathrm{IU} / 1$ in pyogenic meningitis, while Cut-off level of CSF ADA activity is $\geq 5$ in both aseptic \& fungal meningitis. Thus different cut-off levels of CSFADA activities in our experimental studies reveals that there is significant Distinction of differentiation $\quad(\mathrm{p}<0.001) \quad$ between tuberculosis from non-tuberculoses meningitis. There is no significant difference was observed in the ADA levels in CSF in differentiating between aseptic and fungal meningitis, yet the former can easily be differentiated by CSF Cytochemical analysis.

The demonstration of AFB in CSF, CSF culture, CSF Cytochemistry and CT scan are the various tools to diagnose tubercular meningitis, but the ADA estimation in SF is a cost-effective and reliable means to establish a diagnosis of TBM.

In conclusion, $\mathrm{ADA}$ estimation in $\mathrm{CSF}$ is a cost effective, highly sensitive, more specific single test to help a clinician for early and accurate diagnosis of TBM in association with clinical pathological parameters. Also, estimating ADA levels help in early diagnosis and treatment and which in turn helps in reducing the spread of disease.

\section{Acknowledgment}

The authors are grateful to Meril Diagnostics Pvt. Ltd. for giving laboratory facilities and kind permission to publish this paper. 


\section{References}

Blake, J., Berman, P. 1982. The use of adenosine deaminase assays in diagnosis of tuberculosis. S. Afr. Med. J., 62: 19.

Brady, T. Adenosine deaminase. Biochem. J., 36(5): 478 - 484.

Ginsti, G. 1974. Adenosine deaminase in methods of enzyme analysis. Bergmeyer, HV (ed).New York; Academic press, 1092-1099.

Katti, M.K. 2004. Pathogens,treatment and outcome aspects of cerebral tuberculosis, Med. Sci. Monit., 10(9): 215-229.

Malan, C., Donald, D.R., Golden, M. 1984. Adenosine deaminase levels in CSF in the diagnosis of tubercular meningitis.
J. Trop. Med. Hyg., 87(1): 33-40.

Mastroiwni, C.M., Faolette, F., Lichtner, M.D., Agostino, C., Vullo, V., delia, S. 1997. Cerebrospinal fluid cytokines in patients with tuberculous meningitis. Clin. Immunol. Immunopathol., 82(2): 171-176.

Mishra, O.P., Loiwalv Aliz, C.S.F. 1995. ADA and C-reactive protein in tubercular meningitis and partially treated bacterial meningitis. Ind. Pead., 32: 886-889.

Ocana, I., Martinez-Vazquez, J.M., Segura, R.M., Fernandz De-Sevilla, T., Capdevila, J.A. 1983. Adenosine deaminase in pleural fluids. Test for diagnosis of tuberculosis pleural effusion. Chest, 84(1): 51-53.

\section{How to cite this article:}

Goswami, K., S. Mookherjee, K. Sridhar, M. Narayanan and Pal, P. 2016. Comparative Evaluation Studies of CSF ADA Activities in Different Neurological Deficit Groups of Patients with Special Emphasis on Meningitis in Indian Scenario. Int.J.Curr.Microbiol.App.Sci. 5(10): 150-155. doi: http://dx.doi.org/10.20546/ijcmas.2016.510.017 